\title{
Philosophiques
}

\section{Justice distributive et justice rétributive}

\section{Jean-Paul Brodeur}

Volume 24, numéro 1, printemps 1997

Avez-vous lu Rawls ?

URI : https://id.erudit.org/iderudit/027425ar

DOI : https://doi.org/10.7202/027425ar

Aller au sommaire du numéro

\section{Éditeur(s)}

Société de philosophie du Québec

ISSN

0316-2923 (imprimé)

1492-1391 (numérique)

Découvrir la revue

\section{Citer cet article}

Brodeur, J.-P. (1997). Justice distributive et justice rétributive. Philosophiques, 24(1), 71-89. https://doi.org/10.7202/027425ar

\section{Résumé de l'article}

Cet article examine les positions de John Rawls par rapport à la justice retributive (pénale). Nous soutenons d'abord que la perspective de Rawls est éclectique par rapport aux deux polarités exemplaires de la justice pénale soit, en première part, celle du rétributivisme et de l'utilitarisme, et, en seconde part, celle du déontologisme et du conséquentialisme. L'examen des textes révèle que la pensée de Rawls conjugue des éléments qui la qualifient à la fois comme rétributiviste et déontologiste et d'autres qui la rapprochent de l'utilitarisme et du conséquentialisme. Les positions de Rawls sont ensuite scrutées à la lumière des résultats récents de la recherche empirique sur la justice pénale. Nous soutenons alors que la conception rawlsienne de la justice pénale comme un processus sanctionnateur de nature strictement réactive fpost factoj ne peut se concilier avec les aspects pro-actifs de plus en plus affirmés de ce type de justice. On suggère enfin que le principe de Rawls que l'inégalité n'est légitime que lorsqu'elle profite aux plus défavorisés n'a que peu d'application au sein de la justice pénale, dont la cible première est précisément constituée par les pauvres et les sans-pouvoirs.
Ce document est protégé par la loi sur le droit d'auteur. L’utilisation des services d’Érudit (y compris la reproduction) est assujettie à sa politique d'utilisation que vous pouvez consulter en ligne.

https://apropos.erudit.org/fr/usagers/politique-dutilisation/ 


\title{
JUSIICE DISTRIBUTIVE ET JUSTICE RÉTRIBUTIVE
}

\author{
PAR \\ Jenn-Paul Brodeur
}

\begin{abstract}
RÉSUMÉ : Cet article examine les positions de John Rawls par rapport à la justice rétributive (pénale). Nous soutenons d'abord que la perspective de Rawls est éclectique par rapport aux dewx polarités exemplaires de la justice pénale soit, en première part, celle du rétributivisme et de l'utilitarisme, et, en seconde part, celle du déontologisme et du conséquentialisme. L'examen des textes révèle que la pensée de Rawls conjugue des éléments qui la qualifient à la fois comme rétributiviste et déontologiste et d'autres qui la rapprochent de l'utilitarisme et du conséquentialisme. Les positions de Rawls sont ensuite scrutées à la lumière des résultats récents de la recherche empirique sur la justice pénale. Nous soutenons alors que la conception rawlsienne de la justice pénale comme un processus sanctionnateur de nature strictement réactive (post facto) ne peut se concilier avec les aspects pro-actifs de plus en plus affirmés de ce type de justice. On suggère enfin que le principe de Rawls que l'inégalité n'est légitime que lorsqu'elle profite aux plus défavorisés n'a que peu d'application au sein de la justice pénale, dont la cible première est précisément constituée par les pauvres et les sans-pouvoirs.
\end{abstract}

ABSTRACT : This paper explores Rawls' positions on the subject of retributive (criminal) justice. It is first argued that Rawls' perspective is hybrid with respect to the two traditional dichotomies of retributivism us. utilitarianism and of deontologism vs. consequentialism. There is evidence to the effect that Rawls is both a retributivist and a consequentialist. Secondly, Rawls' positions are discussed in the light of recent empirical research. It is argued that his view of retributive justice as being strictly reactive conflicts with the present proactive dimension of criminal justice. It is also suggested that the principle that all inequality should profit the least advantaged members of society has now little application in criminal justice which essentially targets the poor and the powerless.

Dans une étude sur la représentation de la justice dans l'art occidental, Morris Cohen ${ }^{1}$ n'a aucune peine à nous montrer que sa manifestation la plus exemplaire tient dans le jugement qui est rendu

1. Morris L. Cohen, Law : the Art of Justice, New York, Hugh Lauter Levin Associates Inc., 1992. 
par un tribunal dans ce qu'on désigne précisément comme une cour de justice. Or, dans les représentations autant cultivées que populaires, ce jugement est le plus souvent rendu dans le cadre d'affaires pénales où s'incarne la justice rétributive.

La justice rétributive est l'attribution d'un blâme et, dans la plupart des cas, d'une sanction punitive à des personnes qui ont violé une norme. On pourrait dès lors tenter de définir la justice distributive - la justice sociale - de façon inverse. La justice distributive consisterait dans la répartition des avantages selon la conformité des conduites aux normes en vigueur et, de façon plus particulière, aux normes morales. Cette inversion de la définition ne doit pas masquer la solidarité très profonde qui unit justice rétributive et justice distributive. Elle sont en effet définies dans cette perspective de façon rigoureusement symétrique, la seconde étant pour la conformité aux normes ce qu'est la première au regard de leur violation. En outre, ces deux formes de la justice se correspondent ainsi terme à terme inversé dans deux registres en opposition, sans toutefois que l'on soit capable d'établir une relation de préséance de l'un de ces registres sur l'autre.

John Rawls s'insurge contre cette façon de concevoir la relation entre justice rétributive et justice distributive. La justice distributive obéit à une autre logique que celle de la justice rétributive : les avantages sont distribués non pas selon la valeur morale des individus, mais selon leur niveau d'éducation, le besoin qu'ils remplissent et un ensemble d'autres considérations d'ordre socio-économique ${ }^{2}$. Plus profondément, cependant, contrairement à beaucoup de sociologues, Rawls ne pense pas que les manquements à la justice, qui sont sanctionnés de façon rétributive, constituent un aspect permanent (nécessaire) de la vie en socièté ${ }^{3}$. En d'autres termes, on peut concevoir une société idéale où il n'existerait aucune transgression des normes de la justice. Par contre, il est impossible de concevoir une société idéale qui ne soit pas ordonnée par des principes de justice distributive (sociale). Il s'ensuit cette conséquence capitale que la justice rétributive occupe une position subsidiaire par rapport à la justice distributive. Ce rappel de la position subsidiaire de la justice rétributive, alors que nous avons troqué de façon perverse les exigences de la justice distributive (sociale) pour la volonté de punir, confère d'emblêe une actualité à la pensée de Rawls, et sa juste détermination des priorités dans le champ de la justice mérite d'être retenue et approfondie.

Dans $A$ Theory of Justice (TJ). John Rawls ambitionne de produire une théorie des principes fondamentaux de justice, qui pourraient être décrits en langage kantien comme les conditions de possibilité de toute société bien ordonnée. En accord avec sa position sur la primauté de la justice distributive sur la justice rétributive, il devra donc consacrer ses efforts à la découverte et à la formulation des principes de la justice sociale (distributive). C'est effectivement ce

2. Le texte de Rawls sur lequel nous nous appuyons principalement est $A$ Theory of Justice, Cambridge (Mass.). The Belknap Press of Harvard University Press, 1971, seconde édition, 1972, section 49, p. 315.

3. Ibid., section 38 , p. 244-245. 
qu'il fait dans $T J$ et dans la suite de son œuvre. En réalité, $T J$ ne comporte que trois brèves séries de passages portant sur la justice rétributive ou pénale. Dans Political Liberalism, où il approfondit les thèses de $T J$, il n'y a guère qu'un seul passage sur la punition, qui porte sur une question dont l'intérêt est surtout historique (l'existence d'une " loi pénale pure ", chère aux casuistes qui suivaient Suarez ${ }^{4}$ ). Le texte le plus explicite de John Rawls sur la punition date de 1955. Celle-ci n'y figure qu'à un titre ancillaire, n'étant que l'un des tests auquel Rawls soumet sa défense de l'utilitarisme. $e^{5}$ En outre, dans $T J$ et son cuvre postérieure, Rawls ne reprendra pas sa position de 1955 et nous laisse dans l'incertitude quant à la continuité de son adhésion à cette position. Cette incertitude se manifeste clairement chez l'un des rares auteurs qui aient tenté de déduire la position de Rawls sur le droit de punir. Cet auteur, David Hoekema, utilise les principes de $T J$ pour réfuter l'utilitarisme en pénologie en reprenant l'objection classique que l'utilitarisme peut conduire au chátiment des innocents, ce qui serait incompatible avec une certaine lecture de la première partie du Second Principe de la justice ${ }^{6}$. La difficulté à laquelle se heurte la spéculation de Hoekema est que dans son article de 1955, Rawls s'est précisément efforcé de montrer que l'utilitarisme n'était pas vulnérable à cette objection, à la condition de bien comprendre qu'il constituait une justification de la pratique pénale considérée dans son ensemble et non dans ses applications particulières. On ignore s'il soutiendrait toujours cette position dans $T J$ et ses écrits postérieurs à cette œuvre.

Pourquoi, dès lors, consacrer un texte à la position de John Rawls sur la question pénale dans $T J$ et la suite de son œuvre? Nous avons

4. Voir J. Rawls, Political Liberalism, New York, Columbia University Press, 1993 , p. 69.

5. Voir J. Rawls, "Two Concepts of Rules ", The Philosophical Review, vol. 64, $n^{\circ} 1, p .3-33$. Dans ce texte, dont la publication et le contenu coincident avec un article très influent de Anthony guinton ("On Punishment ", Analysis, vol. 14, p. 512-517), Rawls s'efforce de concilier l'utilitarisme et le rétributivisme dans la philosophie pénale, ces deux positions constituant des réponses à deux questions profondement differentes, à savoir le traitement des cas particuliers et l'institution de pratiques générales. On peut en effet se demander pourquoi l'individu $\mathrm{N}$ est puni. On répondra alors de façon rétributiviste en examinant l'action passée de cet individu, faisant alors valoir qu'il mérite une sanction parce qu'il a transgressé une norme légale. On peut toutefois pousser l'enquête plus loin et se demander pourquoi nous possédons quelque chose comme un système pénal parmi nos institutions nos pratiques - sociales. On répondra alors de façon utilitariste en s'appuyant sur les conséquences avantageuses de son existence pour le bien public. Bien que cette position paraisse établir un équilibre entre rétributivisme et utilitarisme en les situant sur des plans divers, Rawls est surtout préoccupé de défendre l'utilitarisme contre les objections les plus courantes qui lui sont opposées comme, par exemple, le fait qu'il autorise la punition des innocents si celle-ci produit des effets sociaux bénéfiques. Sur cette question. voir l'excellent article de Victoria Davion. "Souci et connexion dans l'éthique de la politique générale ", Philosophiques, vol. 22, n ${ }^{\circ} 1$. p. 5363.

6. David A. Hoekema, "The Right to Punish and the Right to be Punished *, dans H. Gene Blocker et Elizabeth H. Smith, John Rawls' Theory of Social Justice, An Introduction, Athens, Ohio University Press, 1980, p. 239-269. Nous confessons une aversion profonde pour les démonstrations du " droit à la punition ", qui constituent l'une des plus aberrantes créations de la philosophie dans le boudoir, pour parler comme le marquis de Sade. 
en partie répondu à cette question lorsque nous avons fait état de la justesse de sa position sur le caractère subsidiaire de la justice rétributive. Cette réponse est toutefois trop génèrale : elle se contente de montrer ce qui s'affiche au fronton de l'œuvre et qui ne justifie pas qu'on veuille y pénétrer plus avant.

De façon plus précise, l'exercice de lecture auquel nous nous livrerons s'autorise de trois raisons. On découvre d'abord des aspects inaperçus d'une œuvre imposante quand on la regarde à travers une fente. Ensuite, la pensée de Rawls sur la justice rétributive, même dans son état inachevé, peut nous servir de stimulant pour réfléchir sur la conjoncture pénale actuelle. Finalement, et peut-ètre de façon plus importante, nous voulons défendre une position philosophique. Nous pensons que la dichotomie entre le fait et la valeur qui surplombe une grande partie de l'éthique ne dégage pas le philosophe moral de sa responsabilité d'avoir une juste appréhension de la réalité historique où s'inscrit son œuvre. C'est un poncif de la philosophie que la science a des comptes à rendre à la morale. Nous soutenons que la réciproque est non moins vraie. La recherche normative ne dispense pas de la connaissance des faits. L'aveuglement complet de ceux qui opposent le caractère sacré de la vie à tout programme de limitation des naissances sur les conséquences cataclysmiques de leur opposition pour les pays les plus pauvres (et les plus vulnérables à des prescriptions désuètes) de la planète est l'une des perversions du normativisme les plus tragiques de notre époque. En conséquence, ce texte aura deux parties, la première consacrée à une brève exégèse de la pensée de Rawls sur la question pénale et la seconde, à une tentative de confronter cette pensée aux pratiques pénales actuelles.

\section{Deux dérivations de la justice pénale}

\section{L. position initiale}

À première vue, il semble à tout le moins que le climat de la pensée pénologique de Rawls soit rétributif. Celui-ci commence en effet par déclarer que $T J$ veut proposer une alternative à l'utilitarisme dans le domaine de la justice distributive. On pourrait s'estimer fondé d'inférer qu'il développerait également une position alternative à l'utilitarisme s'il explicitait davantage sa pensée sur la punition. En outre, les principes de justice pénale qu'il présente dans la section 38 de $T J$, intitulee "The rule of Law", sont des lieux communs du rétributivisme. De façon plus précise, Rawls affirme que le règne de la justice implique que des cas similaires soient traités de manière semblable ${ }^{7}$. C'est là une formulation classique du principe pénal de l'équité selon lequel les mèmes infractions doivent ètre sanctionnées par les mèmes peines. L'utilitarisme a tendance à substituer au principe d'équité le principe d'individualisation des peines. Selon ce dernier principe, la peine doit moins ètre proportionnelle à l'infraction qu'adaptée à l'infracteur et à un pronostic sur sa réinsertion sociale.

7. J. Rawls. A Theory of Justice, section 38, p. 237. 
Il est toutefois une raison plus profonde pour penser que l'horizon de la pensée pénale de Rawls est le rétributivisme. Nous avons déjà commencé à dire que le laconisme de Rawls en matière de justice pénale n'était pas un accident, mais suivait directement de son projet initial. Pour faire vite, on décrira ce projet comme une reprise au niveau de la découverte des principes de la justice sociale du type de démarche que poursuivit Descartes dans sa recherche des premiers principes de la connaissance. Cette démarche se caractérise par une régression vers une situation de doute radical ou d'ignorance, où rien n'est donné et de déduire de cette situation un fondement sur lequel tout le reste pourra s'édifier. Le rapprochement avec Descartes souligne le caractère méthodologique de la démarche de Rawls ${ }^{8}$ : le voile de l'ignorance de Rawls, ainsi que le doute systématique de Descartes, sont des instruments méthodologiques avec lesquels on ne peut construire qu'une situation originelle idéale et, par conséquent, anhistorique.

Dans $T J$, Rawls expose à deux endroits son concept du voile de l'ignorance. La première exposition, au début de l'ouvrage, est rapidement esquissée : là, le voile de l'ignorance se définit par l'ignorance où se trouvent ceux qui déterminent ensemble les conditions originelles du contrat social de leur propre position dans la hiérarchie sociale et des avantages (intelligence, force, fortune et ainsi de suite) dont ils jouiront dans les circonstances effectives de leur existence ${ }^{9}$. Dans la section qui porte explicitement sur le concept du voile de l'ignorance, celui-ci est défini de manière beaucoup plus large. Après avoir défini le voile de l'ignorance de façon similaire à la première caractérisation qui apparaît au début de son livre (l'ignorance de sa propre position sociale et de ses avantages personnels), Rawls ajoute toutefois :

More than this, I assume that the parties do not know the particular circumstances of their own society. That is, they do not know its economic or political situation, or the level of civilization and culture it has been able to achieve. The persons in the original position have no information as to which generation they belong ${ }^{10}$.

Tel qu'il vient d'être défini dans la section de $T J$ explicitement consacrèe à l'exposition de ce concept, le voile de l'ignorance recouvre bien davantage que la situation personnelle de ceux qui se trouvent dans la situation originelle. En fait, ils ignorent tout de la société à laquelle ils appartiendront effectivement. Par son aspect radical, le voile de l'ignorance de Rawls s'apparente effectivement au doute méthodique de Descartes.

La situation originelle où se place Rawls se définit en outre par l'égalité absolue et formelle de tous les sujets, cette égalité autorisant le postulat qu'ils auront tous le même intérêt à respecter les principes

8. C'est là, par exemple, le jugement du sociologue Raymond Boudon : "L'attrait qu'a exercé son livre s'explique peut-être surtout par son aspect le plus solide : sa méthodologie. " R. Boudon, Le juste et le urai Paris, Fayard, 1995, p. 407. Consulter, du même auteur, Effets pervers et ordre social, Paris, P.U.F., 1977, chap. VI.

9. J. Rawls, A Theory of Justice, section 3, p. 12.

10. Ibid., section 24, p. 137. 
fondamentaux de la justice. À cet égard, Rawls distingue entre ce qu'il appelle la théorie de la conformité stricte aux principes de la justice (strict compliance theory) et la théorie de la conformité partielle à ces normes, qui doit expliquer quelle doit être la réponse juste à l'injustice, définie comme transgression des normes de la justice (partial compliance theory) ${ }^{11}$. La préséance de la théorie idéale de la conformitè stricte sur la théorie réaliste de la conformité partielle est fondée de deux façons. La théorie de la conformité partielle repose sur les notions de transgression et de réaction juste à cette transgression. Or, la transgression est essentiellement un concept objectal ou encore transitif : on transgresse quelque chose. L'objet de la transgression est construit à partir des principes et des normes de la justice qui jouissent donc d'une anteriorité logique sur la transgression elle-mēme et qui sont découverts par la théorie idéale de la conformité stricte. A fortiori, pour évaluer le caractère juste de la réponse à l'injustice, on doit disposer de critères qui doivent précéder cette réponse et qui ne peuvent qu'être puisés dans les principes de justice développés par la théorie idéale de la conformité stricte.

Le trait essentiel des pratiques traitées dans le cadre d'une théorie subsidiaire de la conformité partielle est leur caractère réactif. Les matières de cette théorie sont en effet identifiées par Rawls comme étant au premier chef la théorie de la punition, celle de la juste guerre et celle des modes légitimes de résister à un régime injuste (la désobéissance civile, la résistance, l'insurrection armée et la révolution ${ }^{12}$ ). Toutes ces pratiques réagissent à ce qui est perçu comme une injustice. En vertu de son caractère réactif, la punition est tournée vers une action qui lui est antérieure et dont elle constitue la sanction. Ce caractère de la sanction qui consiste à rétablir un équilibre rompu par une transgression passée justifiant son application est le fondement mème du rétributivisme. Celui-ci s'oppose à l'utilitarisme en proposant une légitimation rétrospective de la punition alors que celui-là la justifie de manière prospective par ses effets bénéfiques. Dans un passage de $T J^{13}$, Rawls affirme explicitement que la punition est originellement rétributive et dénonciatrice (primarily retributive or denunciatory). L'établissement d'un lien entre la peine et la dénonciation est caractéristique du rétributivisme.

\section{La première dériva tion de la justice pénale}

Nous allons maintenant tenter de montrer que la position initiale de Rawls entre en conflit avec certains des propos plus explicites qu'il tient sur la justice pénale. Dans $T J$, Rawls nous présente deux dérivations ou deux déductions de la nécessité d'une justice pénale. La première de ces dérivations est la plus importante. Elle ne vise pas spécifiquement la justice pénale dans son sens ètroit, mais la légitimité d'avoir un appareil qui applique de façon coercitive des sanctions contre la transgression des normes du contrat social.

11. Pour ces notions voir ibid., section 2 , p. 8 et les sections 25 et 39.

12. Ibid., section 2 , p. 8 .

13. Ibid., section 38 , p. 241. 
L'emblème de la pensée de Rawls est l'étroitesse du lien qu'il pose entre la justice et l'équité (justice as fairness ${ }^{14}$ ). Cette intrication des deux concepts s'entend de deux manières. D'abord, la situation initiale où les membres potentiels d'une société recherchent les principes de la société bien ordonnée est présentée comme équitable (fair) parce qu'en théorie, personne ne connait encore les circonstances de sa vie et qu'ainsi personne ne risque de faire preuve de partialité en cherchant son avantage particulier dans la définition des principes qui régiront le contrat social ${ }^{15}$.

Elle donne ensuite lieu à la formulation du principe d'équité (principle of fairness). Ce principe affirme que chacune des parties à une entente est requise de faire sa part en conformité avec les règles de l'entente dans l'application de celle-ci, si cette entente remplit deux conditions. La première de ces conditions est que lentente respecte les deux Principes fondamentaux de la justice; la seconde est que la personne qui est partie à l'entente ait accepté volontairement d'en partager les avantages et de jouir des occasions qu'elle lui offre de poursuivre ses intérêts ${ }^{16}$. Ce principe réclame pour l'essentiel que la réciprocité soit la norme fondamentale des échanges par lesquels se réalise la vie en société. Ceux qui se soumettent à des règles qui maximisent la liberté de tous ont le droit d'exiger la même soumission à ces règles de la part de tous ceux qui bénéficient de la soumission des autres. Cette exigence de réciprocité traverse toute l'œuvre de Rawls et elle donne lieu à la première dérivation de la justice rétributive. En effet, le problème qu'il faut résoudre est celui d'offrir aux parties à l'entente une garantie que les autres en respectent effectivement les dispositions, de telle sorte qu'ils ne soient pas minés par le doute que les règles ne sont pas généralement suivies par tous (par exemple, que tous les gens ne paient pas leurs impóts, pour utiliser une illustration chère à Rawls). Il appartiendra à un appareil qui sanctionnera de manière coercitive la non-conformité aux dispositions du pacte de foumir cette garantie :

It is reasonable to assume that even in a well-ordered society, the coercive powers of govermment are to some degree necessary for the stability of social cooperation (...) By enforcing a public system of penalties government removes the ground for thinking that others are not complying with the rules. For this reason alone, a coercive sovereign is presumably always necessary, even though in a well-ordered society sanctions are not severe and may never need to be imposed. Rather, the existence of effective penal machinery senves as men's security to one another. This proposition and the reasoning behind it, we think as Hobbes' thesis ${ }^{17}$.

Ce passage de Rawls est suivi d'une note où il renvoie explicitement au Leviathan (chap. 13-18). Comme la fin du texte le montre, il ne s'agit pas ici d'une référence oblique à la pensée de Hobbes, mais un acquiescement à l'une de ses positions de base.

14. Ces deux concepts ne sont toutefois pas rigoureusement les mèmes. Ibid., section 3, p. 12.

15. Ibid., section 3 , p. 12 .

16. Ibid., section 18, p. 111 .

17. Ibid., section 38 , p. 240 ; voir aussi section 42 , p. $267-268$ et section 49 , p. 315 . 
La machinerie pénale est donc une police d'assurance contre ceux qui veulent bénéficier des avantages du contrat social sans en respecter les contraintes (Rawls les appelle les free riders, ceux qui veulent voyager sans payer). Cette première dérivation de la nécessité des sanctions, de loin la plus significative, n'est pas sans soulever des difficultès.

Quel est, en effet, le ressort de la garantie qui est offerte par l'appareil pénal ? Il est clairement identifié par Hobbes, dont Rawls s'inspire : ce ressort est la crainte du chãtiment (Leviathan, chap. 14). Or, justifier la peine par son effet dissuasif est la caractéristique essentielle de l'utilitarisme et de la pénologie. Notons en outre que la dissuasion est le prototype même de la justification de la peine qui est tournée vers le futur. On ne saurait par définition dissuader quelqu'un de commettre une infraction que cette personne a déjà perpétrée. Cette justification prospective détonne dans l'horizon rétributiviste sur lequel la pensée de Rawls tente de se profiler. Il se peut toutefois que la position défendue par Rawls en 1955 soit effectivement indépassable et que toute justification de l'appareil pénal, par opposition à son fonctionnement dans des cas particuliers, soit de nature utilitariste.

En second lieu, la crainte est un sentiment et, à ce titre, elle est fluctuante. Hobbes prévoit à cet égard qu'un Souverain devra recourir à la terreur pour maintenir la stabilité de son Dominion (Leviathan, chap. 17). La tradition exégétique de la pensée de Hobbes a généralement vu en lui bien plus le père de l'autoritarisme en politique que celui du libéralisme. C'est pourquoi il est gênant de constater que ce qui est désigné comme "la thèse de Hobbes "occupe une place si déterminante dans la pensée de Rawls, qui est l'un des promoteurs du libéralisme les plus en vue.

On notera en dernier lieu que Rawls semble accorder créance à la proposition qui constitue la pierre d'assise de la théorie utilitariste de la dissuasion. Cette proposition soutient que la crainte des châtiments est un des régulateurs les plus opérants de l'agir humain. Cette thèse n'a jamais été validée et l'on possède de très sérieuses indications à l'effet qu'elle est fausse. La question, en réalité, n'est pas tant de déterminer si cette thèse est vraie ou fausse que de souligner que son affirmation simple et indéterminée est presque vide de signification si elle ne s'accompagne pas d'un énoncé des conditions très particulières sous lesquelles la dissuasion est efficace. Il importe au-delà de tout de rappeler que ce n'est pas tant la rigueur du châtiment qui dissuade que la certitude qu'il soit appliqué à tout manquement.

Ces remarques sur la dissuasion nous amènent à traiter brièvement d'une autre des polarités exemplaires de la réflexion sur la justice pénale. Il s'agit de l'opposition entre le déontologisme et le conséquentialisme. Pour le premier, dont Kant est la figure de proue. la peine est entièrement justifiée par la nécessité morale de châtier une infraction aux lois (et également à la morale, si les lois sont justes). Pour le second, représentée de façon éponyme par Jeremy Bentham, la peine doit se justifier tant au regard de la morale qu'au regard du caractère socialement bénéfique de ses conséquences. La position de Rawls par rapport à cette opposition est d'une grande 
subtilité. Sur la foi de son article de 1955 ("Two Concepts of Rule "), on pourrait être tenté d'inférer que Rawls est déontologiste lorsqu'il s'agit de justifier le châtiment d'un infracteur individuel et conséquentialiste lorsqu'il s'agit de justifier l'existence du système pénal. Celui-ci est une institution sociale et sa seule justification ne peut donc résider que dans les conséquences bénéfiques de son existence pour la société.

La distinction claire faite dans l'article de 1955 entre la justification de l'attribution d'une peine à un individu et la justification de l'existence de l'appareil pénal dans son ensemble n'apparaît toutefois plus dans $T J$. C'est pourquoi il est de façon ultime problématique de déterminer quelle est la position de Rawls par rapport aux grandes polarités de la réflexion sur la justice pénale. Au regard de l'opposition entre le rétributivisme et l'utilitarisme, il ne fait pas de doute que ses positions, avec leur accent sur le droit procédural, sont plus proches du rétributivisme. Toutefois, on ne saurait également douter quau regard de la distinction entre le déontologisme et le conséquentialisme Rawls est plus près de la seconde perspective. Le problème que suscite ces prises de position est qu'elles constituent un chiasme. En effet, le rétributivisme est l'une des formes du déontologisme, alors que l'utilitarisme est une des espèces du conséquentialisme. Or, Rawls serait paradoxalement à la fois rétributiviste et conséquentialiste. Cette tension dans sa penseje ne doit pas s'interpréter comme une source de contradictions, mais plutōt comme un effet de distorsion des catégories traditionnelles qui est induit par la complexité des matières afférentes à la justice pénale.

\section{La seconde dériva tion de la justice pénale}

En conformité avec la distinction entre une pénalité (sanction applicable à un délit fiscal, en anglais penalty) et une punition (sanction apportée au crime, en anglais punishment), Rawls distingue parfois entre le simple exercice d'un mode quelconque de coercition pour assurer, par exemple, le paiement des impóts, et l'application du droit pénal stricto sensu. Or, il ne nous offre pas à véritablement parler une démonstration de la nécessité du droit pénal. Ce qu'il nous présente est l'énoncé du principe selon lequel la seule justification qu'il peut y avoir à limiter la liberté individuelle, par exemple, en punissant quelqu'un, est que cette limitation favorise l'épanouissement d'une plus grande liberté collective ${ }^{18}$. Pour le reste, Rawls se contente de suivre H. L. A. Hart et quelques autres théoriciens du droit en rappelant les principes qui doivent régir l'État de droit. Ces principes sont (i) que nul ne saurait exiger d'une personne ce qu'il lui est impossible de faire (ought implies $\operatorname{can}^{19}$ ), (ii) le principe d'équité selon lequel on traitera le semblable de semblable manière ${ }^{20}$, (iii) l'axiome classique Nullum crimen sine $\operatorname{lege}^{21}$ et (iv) l'ensemble des procédures qui préservent l'intégrité du processus judiciaire (due process of (aw) ${ }^{2 \cdot 2}$.

18. Ibid., section 39, p. 244.

19. Ibid., section 38, p. 236.

20. Ibid., section 38, p. 237.

21. Ibid, section 38, p. 238.

22. Ibid. section 38, p. 238-239. 
Non seulement le principe de la limitation de la liberté individuelle au nom de la liberté commune est très général et même à la limite de la banalité, mais il rappelle fâcheusement les pirouettes de certains théoriciens du contrat social qui, comme Jean-Jacques Rousseau, conçoivent la soumission sans bornes au Souverain comme l'obligation d'être libre ${ }^{23}$. Quant aux autres principes qui sont censés ètre à la source de l'État de droit, on les retrouve dans tous les manuels de droit et ils n'ont pas réussi à prévenir l'érosion du système de la justice pénale qui progresse de façon de plus en plus manifeste dans les démocraties occidentales. Pourquoi Rawls réaffirme-t-il des principes dont on sait maintenant à l'usage comment ils sont creux et à quel point ils ont perdu leur vigueur.

La réponse à cette question ne peut prendre la forme que d'une hypothèse qui sera fatalement controversée. On a vu précédemment que la justice rétributive était subsidiaire et que la seule fonction de la coercition, conçue de façon très générale, qui faisait l'objet d'un essai de démonstration est la problématique garantie qu'elle apporte au respect des dispositions du contrat social.

In a well-ordered society there would be no need for the penal law except in so far as the assurance problem made it necessary ${ }^{24}$.

Le droit pénal au sens strict apparait si marginal que Rawls se borne à rappeler les truismes du libéralisme quand il en traite au sens propre. Qu'est-ce qui, encore une fois, peut rendre compte de cette relégation?

On se souviendra que dans le premier passage de Rawls que nous avons précédemment reproduit, il affirme que dans une société bien ordonnèe, les sanctions sont légères et pourraient même n'être jamais appliquées. Pour Rawls, la réaction à l'injustice est reléguée à l'arrière-scène parce que l'injustice n'est elle-même qu'un problème adventice de la vie en société, dont nous avons vu au début de ce texte qu'elle n'en constituait pas un trait permanent. La déviance est conçue dans les termes d'une propension caractérielle ${ }^{25}$ qu'une société bien ordonnée tend à èliminer ou à contrôler ${ }^{26}$. Il s'en suit que dans la société bien ordonnée que Rawls s'efforce de concevoir, le besoin d'un droit pénal, qui répond à l'injustice, est réduit au minimum.

Cette position sur le caractère épiphénoménal de la déviance est aux antipodes de celle de Durkheim pour qui le crime est un trait normal et indépassable de la vie en société.

Imaginez une société de saints, un cloître exemplaire et parfait. Les crimes proprement dits y seront inconnus ; mais les fautes qui paraissent vénielles au vulgaire y soulèveront le mème scandale que fait le délit ordinaire auprès des consciences ordinaires. Si donc cette société se trouve armée du pouvoir

23. Pour Rousseau, toutes les clauses du contrat social se réduisent à une seule, à savoir "l'aliénation totale de chaque associé avec tous ses droits à toute la communauté ", Du contrat social, chap. 6. Rousseau poursuit plus loin en affirmant : "quiconque refusera d'obéir à la volonté générale, y sera contraint par tout le corps; ce qui ne signifie autre chose sinon qu'on le forcera à être libre. "Du contrat social, chap. 7.

24. J. Rawls, A Theory of Justice, section 49, p. 315.

25. Ibid., section 48, p. 315 .

26. Ibid., section 39, p. 245. 
de juger et de punir, elle qualifiera ces actes d'actes criminels et les traitera comme tels ${ }^{27}$.

L'opposition entre Rawls et Durkheim est diamétrale : le pivot de la démonstration de Durkheim est précisément d'affirmer que mème dans une socièté bien ordonnée et parfaite, le crime serait toujours réinventé par ses membres. La raison en est que pour Durkheim le crime est investi d'une fonction sociale positive, qui est de renforcer la solidarité sociale en rassemblant les membres d'une société au sein d'une conscience collective de ce qu'ils condamnent.

Lopposition entre Durkheim et Rawls a toutefois une source beaucoup plus profonde. Pour le second, qui est influencé par les théories du jusnaturalisme, la déviance est une donnée naturelle qui est inhérente à la personnalité du sujet délinquant. Son raisonnement s'oriente à partir du fait de l'injustice et remonte vers la réaction à cette injustice. Durkheim raisonne dans le sens inverse. Pour lui, c'est la peine qui est première et elle construit inlassablement ses objets, qui sont conçus comme des émissaires du renforcement de la solidarité sociale. En un mot, Rawls réfléchit sur le crime, qu'il considère comme une donnée factuelle, alors que Durkheim fait la théorie de la criminalisation, qu'il conçoit comme un processus constructeur de ses objets et comme une dimension indépassable de la réalité de la vie en société, que celle-ci soit envisagée sous le mode idéal ou le mode pratique.

Cette opposition est paradigmatique et dépasse les frontières disciplinaires de la philosophie et de la sociologie. En résumant: 1) Rawls nous invite à penser la peine à partir d'une réaction à la transgression, alors que Durkheim déduit la transgression à partir de la volonté de punir ;2) opérateur de solidarité, le crime est pour Durkheim investi d'une fonction sociale positive qui en constitue la normalité et la pérennité, alors qu'il est versé par Rawls au passif provisoire d'une société, une société totalement solidaire (sans profiteurs) pouvant ètre conçue sans criminalité ; 3) finalement, la pensée de Rawls se penche davantage sur des choses (le crime, la peine) alors que celle de Durkheim reconstruit des processus (criminalisation et pénalisation). Nous ne saurions trancher ces oppositions en faveur de l'un ou l'autre des protagonistes sans outrepasser considérablement les limites de ce texte. Gu'il nous soit toutefois permis de souligner à quel point elles sont exemplaires pour une réflexion sur la justice rétributive.

\section{De la société idéale à la société réalisée}

Les remarques qui clôturent la première partie de ce texte peuvent nous servir d'introduction à cette seconde partie où nous tenterons de confronter la pensée théorique de Rawls à l'épreuve des faits, dans la mesure restreinte où elle peut l'être dans le champ de la justice pénale. Qu'il soit entendu que nous n'allons pas nous limiter à n'utiliser que les éléments incomplets d'une théorie de la justice rétributive que l'on trouve chez Rawls. Ce sont tous les éléments de sa

27. Émile Durkheim, Les règles de la méthode sociologique, Paris, P.U.F., 1981, p. 68 . 
théorie de la justice que nous estimons susceptibles d'une interrogation, dans la mesure où ils sont en prise avec la conjoncture actuelle de la justice pénale.

\section{Les modes divers de la coercition}

Nos premières remarques porterons sur les éléments d'une théorie de la justice rétributive (pénale) que l'on trouve chez Rawls. Cette attribution personnelle est en réalitè problématique, car l'attitude que nous allons questionner est celle de la majorité des philosophes, qui s'accordent avec Foucault lorsqu'il constate que l'on n'a pas beaucoup réfléchi jusqu'à aujourd'hui sur le pouvoir et son exercice. La dérivation de la justice pénale repose chez Rawls, comme chez Hobbes et les autres théoriciens du contrat social, sur une perception de la nécessité d'un appareil coercitif pour sanctionner les écarts. La coercition est pour l'essentiel conçue post facto comme une réaction à la transgression. Elle peut ètre investie d'une fonction préventive, mais cette fonction s'exerce moins par son exercice effectif que par la menace de son application. Cette conception de la coercition/réaction est un lieu commun de la philosophie politique. Elle est malheureusement très incomplète.

En effet, une grande partie des crimes ne sont pas des mesures de rétorsion, mais des actes de pure agression. On s'étonne qu'on n'ait pas appliqué cette constatation élémentaire aux actions de l'État qui, pour une grande partie, ne sont que le décalque public des comportements privés. Le tableau suivant est construit à partir d'une démarcation entre deux dimensions absolument distinctes de la répression pénale, à savoir l'exercice proactif de la coercition, simplement désigné dans notre tableau comme coercition, et son exercice réactif, identifié comme sanction. Cette distinction permet la construction de quatre cas de figure, selon la présence ou l'absence de l'un des deux termes de la distinction.

\begin{tabular}{|c|c|}
\hline COERCITION & SANCTION \\
\hline \begin{tabular}{l}
\multicolumn{1}{c}{+} \\
Test d'alcoométrie \\
Détecteur de métal \\
Fouille \\
Perception des impoots \\
Prévention du crime \\
et de la déviance
\end{tabular} & \begin{tabular}{l}
\multicolumn{1}{c}{+} \\
Peine pour ivresse au volant \\
Peine pour possession d'arme \\
Peine pour possession de substance p. \\
Peine pour fraude fiscale
\end{tabular} \\
\hline $\begin{array}{l}\text { Quarantaine médicale } \\
\text { Extorsion (comuption) } \\
\text { Gestion des sans-abris }\end{array}$ & - \\
\hline Chiffre noir de la déviance & $\stackrel{+}{+}$ \\
\hline $\begin{array}{c}\text { - } \\
\text { Tolèrance : drogue douce } \\
\text { prostitution }\end{array}$ & - \\
\hline
\end{tabular}

Le premier cas de figure représenté dans ce tableau est celui de la présence simultanée de coercition et de sanction. La perception des impóts, pour reprendre un exemple cher à Rawls, s'est effectuée jusqu'à la fin du XVIII de manière coercitive, comme l'ont remarqué 
un grand nombre d'historiens des mœurs (par exemple, Norbert Elias ou Egon Bittner). On ne se contentait pas, en effet, de réprimer la fraude fiscale. La perception mème des impōts était effectuée par des gens d'armes contre la volonté des contribuables (en majorité des paysans) qui percevaient cette collecte comme une agression du pouvoir à laquelle ils résistaient. La sanction proprement dite s'ajoutait à la coercition pour les contribuables qui résistaient trop. Un exemple plus contemporain est l'établissement de barrages routiers où les conducteurs de véhicules automobiles sont contraints de subir des alcootests. $\dot{A}$ ce premier exercice de la contrainte s'ajoute la sanction pénale imposée à ceux dont le taux d'alcoométrie dépasse celui qui est autorisé par la loi.

À partir de la discussion de ce premier cas de figure, les autres cas sont faciles à présenter. Le second cas se caractérise par la présence de coercition et par l'absence de sanction. Il s'agit, pour la majorité, de cas où des personnes font lobjet de mesures coercitives non pas en vertu de ce qu'elles ont fait - une transgression des normes - , mais en vertu de ce qu'elles sont (malades, économiquement vulnérables, sans ressources). Le troisième cas met en contraste les cas de tous ceux qui commettent impunément des infractions (le chiffre noir de la délinquance) et de ceux qui ont été découverts, arraisonnés et sanctionnés. Il est à cet égard des types de délinquance - en particulier la délinquance économique - qui se produisent généralement sans interférence de la coercition et de la sanction publiques. Il arrive toutefois que l'État cède à la pression publique et sanctionne de manière exemplaire la délinquance économique (par exemple, dans le cadre de campagnes contre la corruption). Le dernier cas de figure se caractérise par l'absence relative de coercition ou de sanction. Il se distingue du précédent en ce que les actions commises se produisent au grand jour, bien qu'elles soient prohibées. Elles font l'objet d'une tolérance, qui n'est pas sans impliquer tout un ensemble officieux de mesures de harcèlement et, par conséquent, de coercition (pensons ici à la prostitution).

Ce tableau est bien incomplet. Une enquête systématique devrait distinguer entre des mesures de coercition préventives lle détecteur de métal), des cas où coercition et délinquance se produisent concurremment (un affrontement entre manifestants et forces de l'ordre) et enfin l'exercice post-factum des sanctions pénales. En outre, il faudrait tenir compte du taux de visibilité des comportements visès par la répression pénale dans ses trois dimensions temporelles. Ainsi, si l'on exige un âge déterminé comme condition d'entrée dans un service, il est à prévoir que beaucoup de personnes mentiront sur leur āge, personne ne portant son àge sur son visage ni mème dans son corps, à quelques années près. Cependant, si l'on exige une taille déterminée (par exemple, $1 \mathrm{~m} \mathrm{75}$ ), il serait déraisonnable pour une personne de tenter de transgresser la norme puisque cette tentative serait immédiatement apparente.

En dépit néanmoins de son caractère incomplet, le tableau que nous avons esquissé est suffisant, nous l'espérons, pour montrer à quel point il est sommaire de traiter de la coercition exclusivement sous son mode réactif. 


\section{Égalité et dis crim ina tion}

Le second des principes fondamentaux de la justice énoncés par Rawls comporte deux parties, la première de celles-ci étant connue comme le Principe de différence. Ce principe affirme essentiellement que toute forme d'inégalité n'est admissible que dans la mesure où elle profite aux plus désavantagés ${ }^{28}$. Ce principe a généré beaucoup de discussion. Récemment, des chercheurs américains ont construit sur papier quatre situations distinctes au niveau de la répartition des niveaux de rémunération (niveaux des salaires), de manière à illustrer quatre principes différents de justice distributive. Lun de ces principes, ainsi que la situation qui l'illustre, correspondait au principe de différence de Rawls. Traduit dans un schème de détermination des niveaux de rémunération, le principe de différence exigeait la " maximisation " du salaire minimum. Ces situations illustrant quatre principes différents de justice sociale ont été soumis à 81 groupes expérimentaux, aux États-Unis et en Pologne, dont les sujets devaient donner leur adhésion à celle qui leur paraissait la plus juste. La situation qui a recueilli le plus haut taux d'adhésion $(77,8 \%)$ correspondait à une "maximisation "du salaire moyen avec une contrainte pour le salaire minimum, dont le niveau devait ètre acceptable. La situation correspondant au principe de différence de Rawls est celle qui, avec un taux de $1,23 \%$, a recueilli le moins d'adhésion parmi les quatre principes sélectionnés pour l'expérience $^{29}$.

Pour intéressants que soient ces tests empiriques, ils peuvent également ètre contestès comme peut l'ètre tout résultat d'une recherche empirique. Nous aimerions soumettre le principe de différence de Rawls à un autre type de validation empirique, de nature moins expérimentale. Le principe de différence exige que les inégalités profitent aux plus désavantagés. Ce principe implique que l'égalitê est en elle-même naturellement juste et qu'il n'en résulte pas une situation problématique qui devrait être soumise à un principe de justice. En d'autre termes, c'est l'inégalité qui fait problème pour Rawls et les théoriciens du contrat social, et non pas l'égalité. Dans une conjoncture rigoureusement égalitaire, la question des plus désavantagés ne se poserait en théorie même pas. Or, il y a lieu d'interroger ce postulat théorique à la lumière de la situation actuelle des Afro-Américains.

Voici un rapide portrait statistique de leur situation ${ }^{30}$ :

1. Entre 1980 et 1990, la population carcérale des États-Unis a triplé. On évalue maintenant qu'entre 1,2 et 1,6 millions de personnes sont incarcérées, selon que l'on tient compte ou non de la détention préventive avant procès. Lorsque la population carcérale américaine atteindra 2 millions de personnes, près de $1 \%(0,77 \%)$ de la population américaine sera sous les verrous.

28. J. Rawls, A Theory of Justice, section 13, p. 83.

29. Voir N. Frohlich et J. A. Oppenheimer, Choosing justice, an Experimental Approach to Ethical theory. Oxford, University of California Press.

30. Ces statistiques proviennent de diverses sources dont la principale est Michael Tonry, Malign Neglect: Race, Crime and Punishment in America, New York, Oxford University Press, 1995. 
2. Les Afro-Américains formaient, en 1992, $13 \%$ de la population américaine et $54 \%$ de la population carcérale.

3. De 1979 à 1993, le pourcentage des Afro-Américains incarcérés est passé de $39 \%$ à $54 \%$. Il s'agit d'une progression fulgurante, au regard de l'augmentation elle-mème rapide de l'incarcêration.

4. Le taux d'incarcération aux États-Unis s'élève maintenant à 519 personnes par 100000 habitants. Il est le plus élevé des démocraties occidentales. Par comparaison, le taux d'incarcération au Canada, qui se place au second rang parmi les démocraties occidentales, fluctue autour de 118 personnes par 100,000 habitants.

5. En 1991, aux États-Unis, le taux d'incarcération des Noirs était de 1895/100 000, alors que celui de la population blanche se situait à 293/100 000, c'est-à-dire que ce dernier ètait 6 fois moins élevé.

6. Aux États-Unis, $23 \%$ des Afro-Américains de sexe masculin sont sous la coupe du système pénal (incarcérés ou sous surveillance). Cette proportion est de $33 \%$ dans l'État de Californie. En 1991, $42 \%$ des hommes Afro-Américains àgés entre 18 et 35 ans ètaient sous le joug du système pénal dans la juridiction de Washington D.C. Cette proportion s'élève mème à $56 \%$ à Baltimore.

7. Dans les prisons fédérales américaines, la proportion des détenu(e)s incarcéré(e)s pour une infraction en matière de drogues est passée de $25 \%$ (1980) à $58 \%$ (1992).

8. Dans l'État de Pennsylvanie, qui n'est en rien atypique, les incarcérations pour délits en matière de drogue ont augmenté de $1613 \%$ pour les non-Blancs et de $477 \%$ pour les Blancs, de 1980 à 1990.

Ces chiffres nous présentent un tableau relativement effarant de la disparité qui existe entre les taux d'incarcération des AfroAméricains et ceux de la population blanche. Les chercheurs américains se sont penchés sur cette situation pour en découvrir l'explication. Le facteur déterminant réside dans la croisade anti-drogue lancée par le président Reagan et qui s'est considérablement amplifiée sous les présidents Bush et Clinton. Comme on peut le constater en consultant les chiffres cités plus haut, cette croisade a frappé la population afro-américaine de façon absolument disproportionnèe, alors que les Afro-Américains ne consomment dans l'ensemble pas plus de drogues que la population blanche.

Le problème est qu'ils consomment autrement. Depuis le début des années 1980, les Américains ont voulu combattre les disparités, en grande partie raciales, dans l'imposition des peines. Beaucoup d'États ont donc promulgué ce qu'on appelle des lignes directrices en matière de détermination des peines ${ }^{31}$. Essentiellement, ces lignes directrices proposent aux magistrats américains des formules pour calculer la nature et la durée d'une sanction pénale en se basant, par

31. Pour mieux connaitre la nature de ces lignes directrices, voir Commission canadienne sur la détermination de la peine, Réformer la sentence: une approche canadienne. Rapport de la Commission canadienne sur la détermination de la peine, Ottawa. ministère des Approvisionnements et Services Canada, février 1987. Voir également Jean-Paul Brodeur. "Réforme pénale et sentences : expériences nord-américaines ", Déviance et Société, 1985, vol. $9, n^{\circ} 3$, p. 165-200. 
exemple, sur la gravité de l'infraction et le dossier judiciaire antécédent de l'infracteur(e).

Ces lignes directrices peuvent toutefois être beaucoup plus détaillées et donner comme instructions aux juges de prendre en considération la nature de la substance en cause dans des affaires de drogue. Un exemple notoire et extrèmement influent a été fourni par les lignes directrices élaborées par le gouvernement central américain à l'intention des cours fédérales (plusieurs États se sont alignés sur ces lignes directrices). La commission qui a développé ces lignes à partir du milieu des années 1980 a estimé que la cocaïne présentait sous la forme de cristaux de crack un danger beaucoup plus grand que sous la forme de poudre. L'article $2 \mathrm{D} 1.1$ de ces lignes directrices précise qu'il faut multiplier par un facteur de 100 les quantités de crack impliquées dans une cause de drogue et imposer les peines en fonction de cette multiplication. En pratique, le juge doit punir une personne en possession de 10 grammes de crack (ce qui est une très petite quantité de drogue) de la même manière qu'une personne en possession d'un kilo de cocaïne ou d'héroïne en poudre (ce qui est une quantité considérable). Cette règle a pour effet de transformer toutes les causes de possession simple de drogue, qui appellent des sanctions légères, en des causes de trafic de drogue, qui réclament des sanctions très lourdes. Par exemple, dans l'État de Minnesota, pourtant l'un des plus libéraux des États-Unis, la possession de 3 grammes de crack est sanctionnée par une sentence minimale de 4 ans de prison, cette sentence pouvant s'élever jusqu'à un maximum de 20 ans d'incarcération ; par contraste, la possession de 3 grammes de poudre de cocaïne n'entraine de façon obligatoire qu'une peine de probation, avec la possibilité d'alourdir cette peine jusqu'à un maximum de 5 ans d'incarcération. Les différences dans la sévérité des peines sont considérables, la possession de 3 grammes de crack entrainant automatiquement une peine d'au moins 4 ans de prison, alors que la possession de la même quantité de cocaïne sous la forme de poudre n'est dans la majorité des cas même pas soumise à l'incarcération.

Comme la population afro-américaine est beaucoup plus encline à consommer du crack - moins cher et plus puissant que la poudre alors que les Blancs se droguent avec de la cocaïne et de l'héroïne en poudre, il n'est pas étonnant que l'application de ces lignes directrices en matière de détermination de la peine ait eu pour résultat l'incarcération massive de contrevenants afro-américains. Or, il était pleinement connu des législateurs américains que c'étaient les AfroAméricains qui étaient les premiers consommateurs de crack et que la multiplication par 100 des quantités de crack saisies aux fins de détermination de la peine allait entrainer une poussée irrésistible de l'incarcération des membres de cette minorité. Au cours de nos recherches, nous avons tenté de savoir quelle place cette prévision du résultat des lignes directrices fédérales avait tenu dans leur élaboration. La réponse qu'on nous a fournie ${ }^{32}$ rappelle étrangement

32. Nous avons en particulier interrogé M. Philippe Heyman, qui était assistantprocureur général des États-Unis, c'est à dire le commandant en second du ministère américain de la Justice. 
les positions de Rawls. Essentiellement, la commission qui a èlaboré ces lignes directrices s'est volontairement placée sous le voile de l'ignorance afin de préserver l'impartialitê de son jugement, en respectant de façon absolue l'égalitê formelle de tous devant la loi. Cette position, irréprochable au niveau des principes, a eu des résultats discriminatoires catastrophiques au niveau de la pratique. Avec le recul, ces résultats sont maintenant décrits comme prévisibles bien qu'on se soit par principe aveuglé sur leur prévisibilité -, mais non intentionnels.

On savait ce que l'on faisait, tout en affirmant n'en pas vouloir les résultats. Cette dissociation entre le savoir et l'intention est intenable en droit, mème si on peut de façon méthodologique l'effectuer au plan de la théorie. C'est comme si, au nom du droit égal de tous au traitement médical, on injectait le mème remède à une population malade tout en sachant qu'un pourcentage aisément repérable de cette population était allergique au traitement et n'y survivrait pas. Pourrait-on alors se défendre en déclarant avoir agi pour le bien du plus grand nombre et que la mort des personnes allergiques ètait un résultat prévisible, mais non intentionnel de l'application? Il est possible qu'on soit absout des résultats de son action par le tribunal idéal de la philosophie. Il est très improbable qu'on jouirait d'une telle faveur devant une véritable cour de justice.

La question qui a donné naissance à ce texte, d'abord présenté sous la forme d'une communication orale, était "Pourquoi faut-il lire Rawls ? . Il est une première façon de répondre à cette question, de façon positive. Nous avons vu que Rawls maintenait non seulement que justice rétributive et justice distributive n'obéissaient pas à une même logique, mais que la seconde avait préséance sur la première. Nous pensons que cette détermination des priorités constitue à l'heure actuelle un antidote dont nous avons grand besoin contre le refoulement partout sensible de la justice sociale au profit de la justice pénale, qui fait maintenant l'objet d'un engouement frisant l'hystérie.

Nous pouvons ensuite fournir une réponse interrogative à cette question. Dans son article de 1955, Rawls nous propose la définition suivante de l'institution de la punition ${ }^{33}$ :

I begin by defining the institution of punishment as follows : a person is said to suffer punishment whenever he is legally deprived of some of the nomal rights of a citizen on the ground that he has vilated the rule of law, the violation having been established by trial according to the due process of law, provided that the deprivation is carried out by the recognized legal authorities of the state, that the rule of law clearly specifies both the offense and the attached penalty. that the court construe statutes strictly and that the statute was on the books prior to the time of the offense.

Cette définition est en tous points ètonnante. Elle est d'abord complètement normative, ne définissant pas la punition - les termes de mal, de douleur, de tort ou de préjudice n'apparaissant nulle part dans la définition --, mais déterminant les conditions idéales de son 
application. Le paradoxe de cette définition est qu'elle ne trouve aucune application dans les sociétés les plus punitives, où la plupart des conditions énoncées par la définition ne sont pas respectées (c'est d'ailleurs pourquoi ces sociétés sont si punitives). En second lieu, cette définition est essentiellement procédurale, ce qui est une autre façon de répéter sa première caractéristique : c'est moins la punition qui est définie que la procédure qui doit être suivie dans son application. Finalement, cette définition est profondément ethnocentrique à un degré qui étonne : elle ne s'applique qu'aux démocraties occidentales pour la période commençant avec la fin du XIX siècle.

Il ne semble pas que John Rawls ait jamais varié sa position sur l'aspect formel ou procédural du droit pénal. La section de $T J$ qui est la plus explicite sur la justice rétributive est la section 38 , intitulée "The rule of law". Or, le droit pénal se divise grosso modo en droit substantiel et en droit de la procédure. Il n'est pas sūr que si Rawls avait considéré autant le droit pénal substantiel que les normes de la procédure, il aurait pu ètablir une distinction aussi tranchée entre les normes de la justice distributive (sociale) et celles de la justice rétributive (pénale). En effet, on pourrait soutenir que la prohibition du meurtre, du vol ou du viol ne sont qu'une autre mise en forme du droit à la vie, à la proprièté et à l'intégrité physique de sa personne. La réciprocité de la justice distributive et de la justice rètributive s'affirme d'une façon beaucoup plus ferme dès que l'on quitte les normes procédurales du droit pénal pour examiner les normes substantielles qu'il propose et dont le lien avec la morale est, pour les plus récurrentes d'entre elles, très étroit.

Nous proposerons enfin une réponse plus critique et, au vrai, plus négative. L'entreprise de Rawls nous rend sceptique à un double ègard. D'abord par sa forme, qui est celle de la synthèse déductive propre aux systèmes de métaphysique du rationalisme et de l'époque des Lumières. Le renoncement de Wittgenstein à l'expression systématique de la pensée philosophique, après le Tractatus, nous paraìt à cet égard exemplaire : il n'est pas súr que les grandes synthèses à la Rawls soient beaucoup plus qu'un puissant exercice de l'esprit qui cependant ne réussit pas à ètre en prise de façon significative sur la réalité. Ensuite, il nous semble clair que dans le débat sur la pertinence de réaffirmer des positions élaborées essentiellement à l'époque des Lumières pour interpréter la réalité contemporaine et pour la faire évoluer vers un ètat meilleur, Rawls se situe du cōté de penseurs comme Jürgen Habermas ou Charles Taylor. Ceux-ci estiment, avec beaucoup d'autres de moindre envergure, qu'un retour s'impose aux valeurs et aux thèses defendues par les penseurs des Lumières. Dans le champ limité qui est le nōtre, celui des études sur la déviance et celui de la justice pénale, nous différons complètement d'opinion. Le monde de la justice pénale est résolument post-moderne et nous ne voyons pas comment, par exemple, des instruments conceptuels qui ont contribué au développement d'une justice procédurale fondée sur les droits individuels dans leur définition technico-juridique peuvent ètre encore exploités pour conduire une réflexion sur une justice de masse qui est en train de s'effondrer sous le volume des affaires qu'elle recueille et dont les procédures sont en train de se métamorphoser sous l'assaut des médias. Tenter de 
montrer que la justice pénale est entrée dans un au-delà de la modernité, telle qu'elle s'est définie depuis le rationalisme du XVI ${ }^{e}$ siècle, excède évidemment le propos de ce texte qui, avec cette dernière phrase, est parvenu à son terme.

École de criminologie

Centre international de criminologie comparée Université de Montréal 


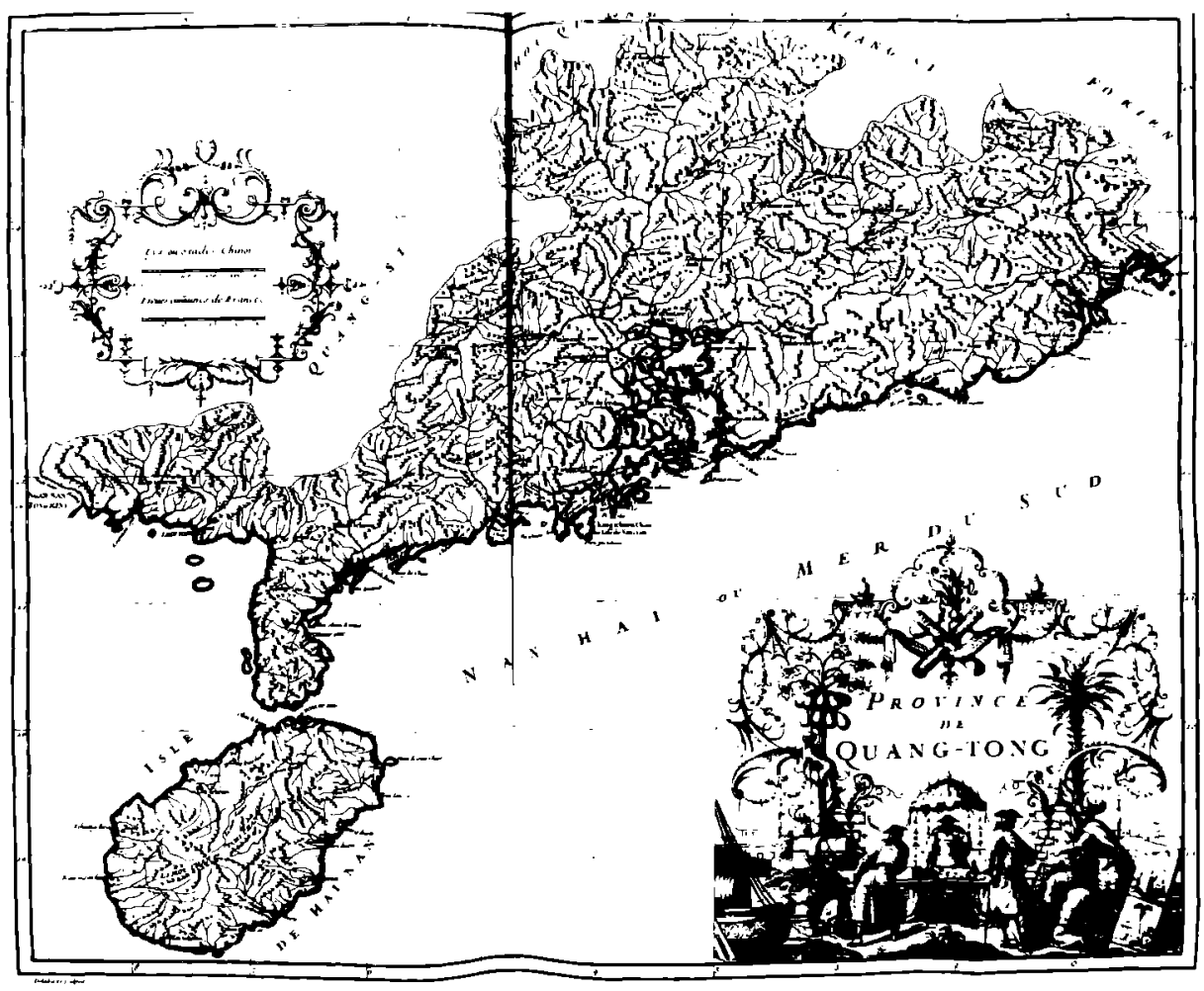

Description géographique, historique, chronologique, politique et physique de l'Empire de la Chine et de la Tartarie chinoise, par le P. J.-B. du Halde, de la Compagnie de Jésus, tome I, à Paris, chez P. G. Le Mercier, Imprimeur-Libraire, rue Saint Jacques, au Livre d'Or, M.DCC.XXXV.

Nîmes, Carré d'Art Bibliothèque, cote 12133/1 